\title{
Ultra-Wide Band Diversity Antenna for Omnidirectional Coverage
}

\author{
Maxime Volery ${ }^{1}$, Tatjana Asenov ${ }^{1}$, Anja K. Skrivervik ${ }^{1}$ \\ ${ }^{1}$ Microwaves and Antennas Group (MAG), Ecole Polytechnique Fédérale de Lausanne, Lausanne, Switzerland \\ tatjana.asenov@epfl.ch
}

\begin{abstract}
In this paper, a $41 \times 42.2 \mathrm{~mm}^{2}$ ultra-wide band (UWB) diversity antenna is designed to operate in the frequency band 5.45- 7.45 $\mathrm{GHz}$ with the isolation better than $15 \mathrm{~dB}$. To achieve omnidirectional coverage pattern diversity is applied. The proposed design is suitable for UWB applications requiring reliable radio link and coverage.
\end{abstract}

Index Terms - UWB, pattern diversity, omnidirectional coverage.

\section{INTRODUCTION}

The significant growth of data traffic underlines the need for concepts allowing high data rates, better coverage and reliable services. The release of $3.1-10.6 \mathrm{GHz}$ frequency spectrum for unlicensed usage in 2002 has sparked interest in Ultra-Wideband Technology. This technology is characterised by large bandwidth, low power consumption, short-range high data rate and low cost that have been exploited for various applications, e.g. commercial wireless applications, medical applications, consumer electronics etc. [1-3]. However, UWB systems can be prone to multipath fading [2]. To mitigate multipath fading different diversity techniques, such as spatial, polarization or pattern diversity, can be applied [4-5].

In this paper, an UWB diversity antenna with omnidirectional pattern is presented. The proposed antenna is suitable for UWB applications that require a reliable radio link and an omnidirectional coverage.

\section{DESIGN CONCEPT}

The designed antenna consists of two different radiating elements placed on a substrate FR-4 characterised by a dielectric constant of 4.4 and a loss tangent of 0.018 . The substrate has the thickness of $1.6 \mathrm{~mm}$ while the dimensions of the proposed antenna are $41 \times 42.2 \mathrm{~mm}^{2}$. The microstrip lines are designed to ensure $50-\Omega$ feeding. Since the second antenna is placed at the angle of $90^{\circ}$, to avoid reflection a mitered band is designed [6] A detailed antenna geometry is illustrated in Fig. 1 and Fig. 2.

Pattern diversity has been applied to ensure coverage without blind spots. This diversity technique is based on combining multiple directional antenna with different radiation patterns. Furthermore, there are various ways of combining antennas, e.g. based on signal-to-noise ratio (SNR) after maximal ratio, equal gain or selection diversity [3]. In this paper, selection diversity is used to assess the coverage and radiation pattern of the proposed UWB diversity antenna. This diversity concept is based on selecting the antenna with the highest SNR. Hence, only one antenna will be in use at the time.

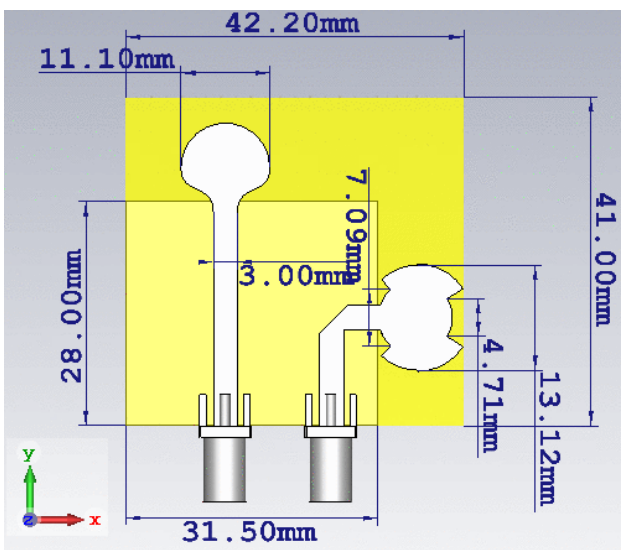

Fig. 1 Top view of the antenna geometry

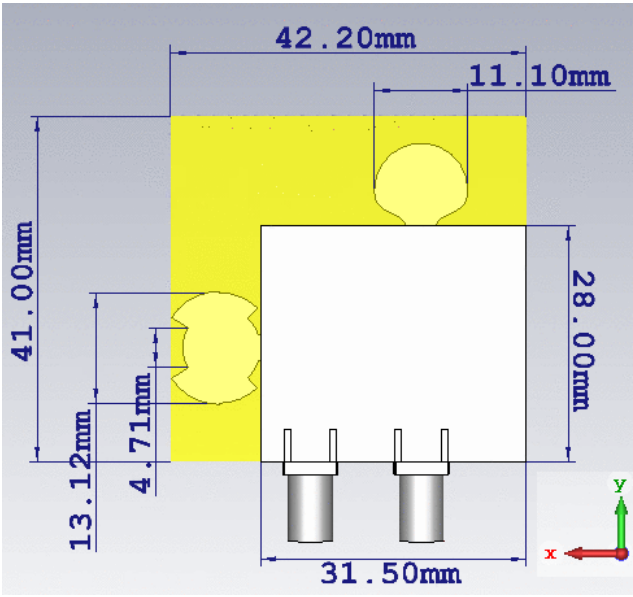

Fig. 2 Back view of the antenna geometry 


\section{RESULTS AND DISCUSSION}

CST Microwave Studio has been used to study and design the proposed UWB diversity antenna. The simulated Sparameters results are given in Fig.3. The measurement results will be presented at the conference.

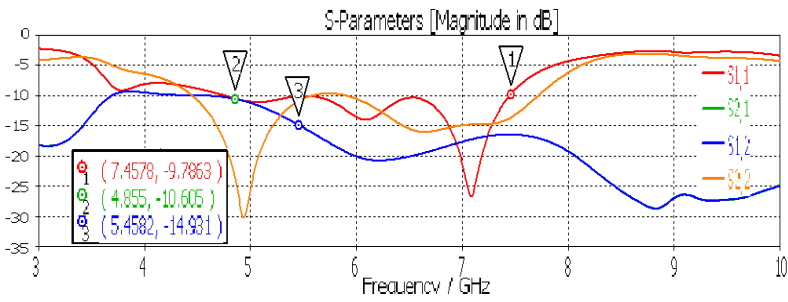

Fig.3 Simulated S-parameters of the proposed UWB diversity antenna

As it can be seen, the reflection coefficients of antennas are less than $-10 \mathrm{~dB}$ in the following frequency bands, i.e. $\mathrm{S} 11<-10 \mathrm{~dB}$ in the $4.75-7.45 \mathrm{GHz}$ frequency band while $\mathrm{S} 22<-10 \mathrm{~dB}$ in the $4.45-7.75 \mathrm{GHz}$ frequency band. However, mutual coupling between two ports is less than $-15 \mathrm{~dB}$ in the 5.45-10 GHz frequency band. Therefore, good performance of the antenna is expected in in the $5.45-7.45 \mathrm{GHz}$ frequency band.

The far field patterns of realized gain at different frequencies shown in Fig. 4-6, are obtained when ports were excited simultaneously, i.e. when one port is excited the other is matched to $50-\Omega$ load. The radiation patterns are generated at $6.4 \mathrm{GHz}, 6.8 \mathrm{GHz}$ and $7.2 \mathrm{GHz}$.

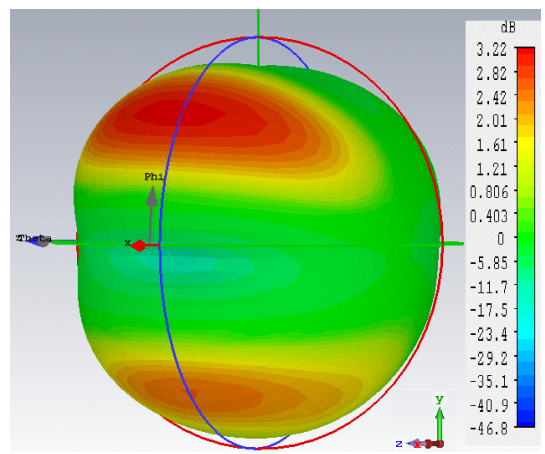

a)

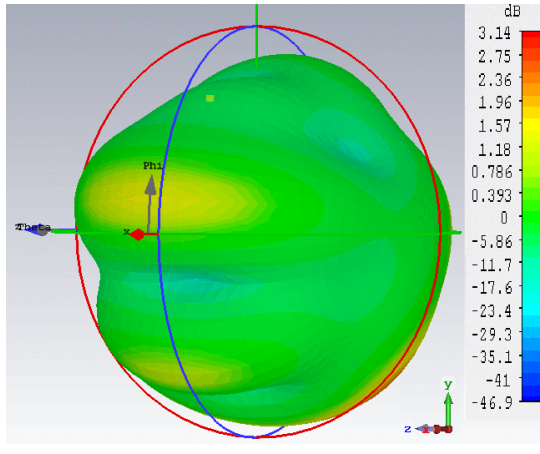

b)

Fig. 4 a) port 2 is matched at $6.4 \mathrm{GHz}$, b) port 1 is matched at $6.4 \mathrm{GHz}$

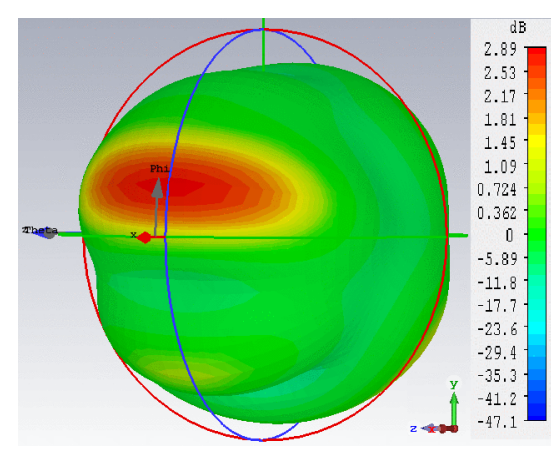

a)

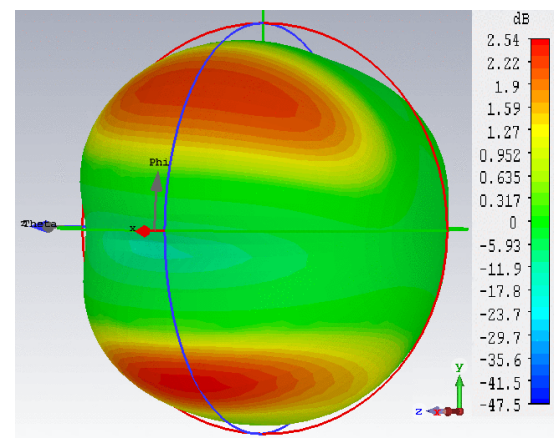

b)

Fig. 5 a) port 2 is matched at $6.8 \mathrm{GHz}$ b) port 1 is matched at $6.8 \mathrm{GHz}$ 


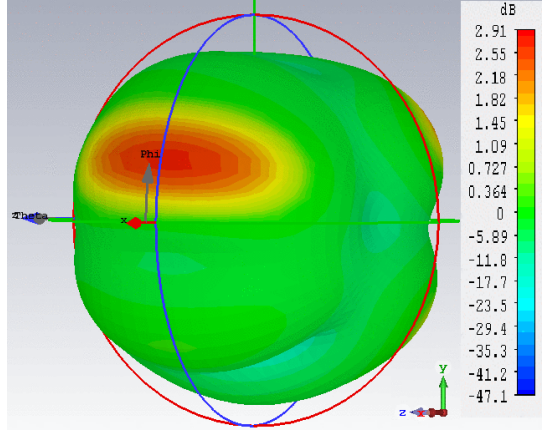

a)

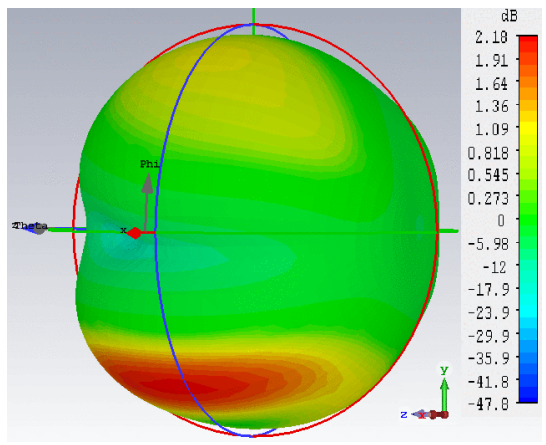

b)

Fig. 6 a) port 2 is matched at $7.2 \mathrm{GHz}$ b) port 1 is matched at $6.8 \mathrm{GHz}$

To estimate and analyse coverage, gain and directivity of the UWB diversity antenna a selection diversity technique has been implemented in a MATLAB code. The combining gain and directivity represent the maximum value of gains and directivities of both antennas in each direction. Furthermore, deviation from maximum gain less than $10 \mathrm{~dB}$ is estimated to be enough to ensure good coverage, i.e. to avoid pattern nulls. Subsequently, the percentage of all directions where deviation from maximum gain is higher than $10 \mathrm{~dB}$ has been calculated. The obtained results are given in Fig.7.
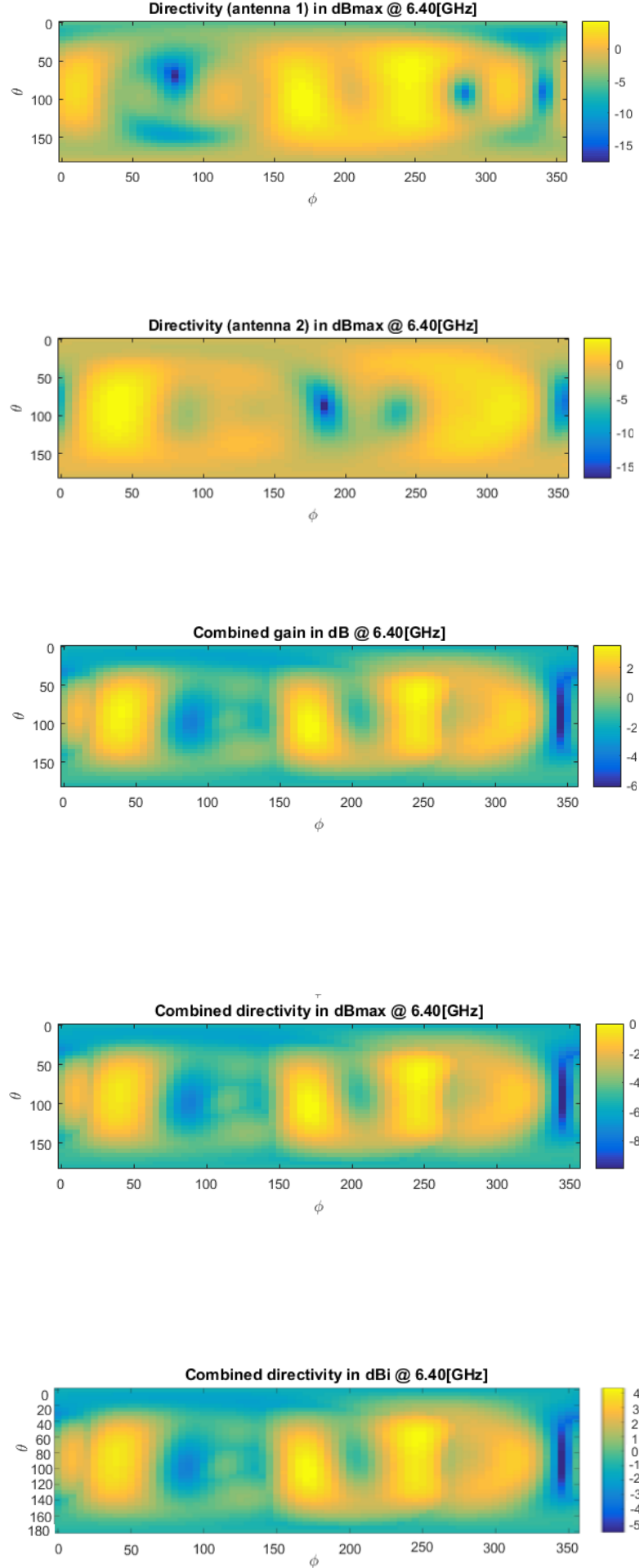

a) 

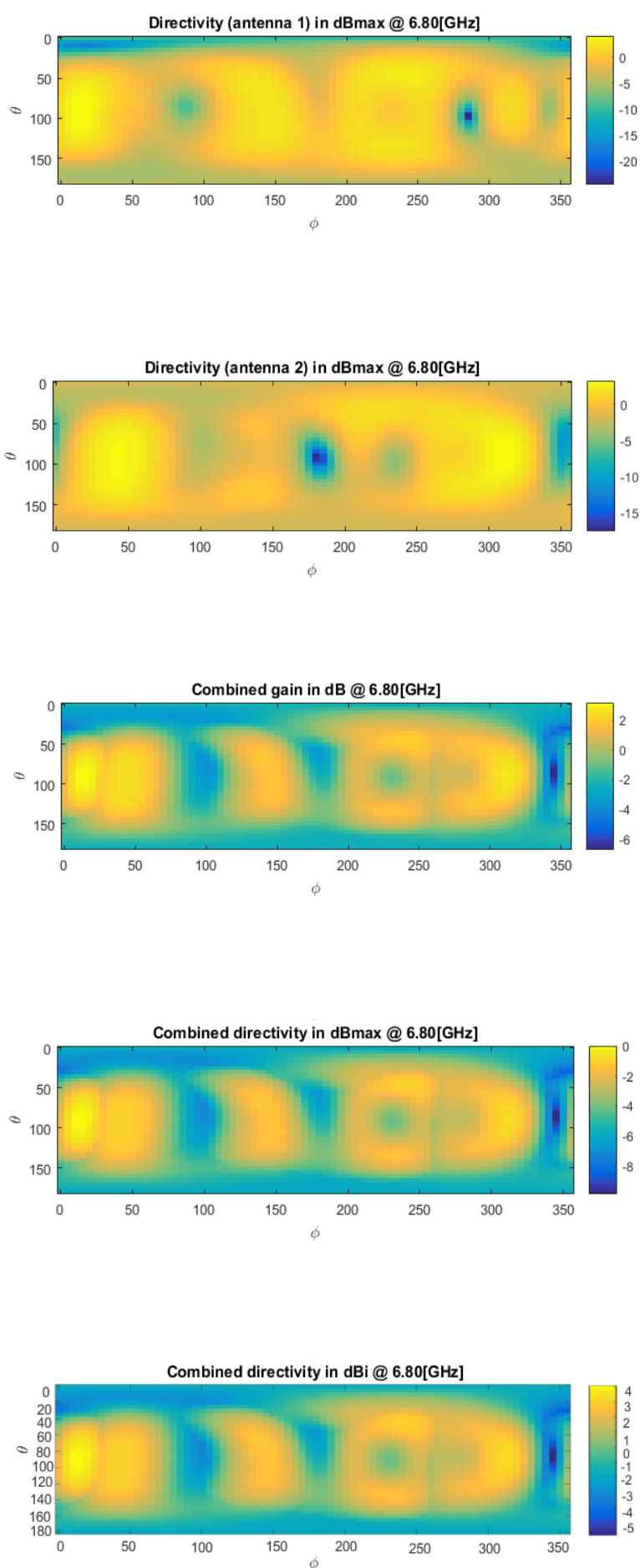

b)
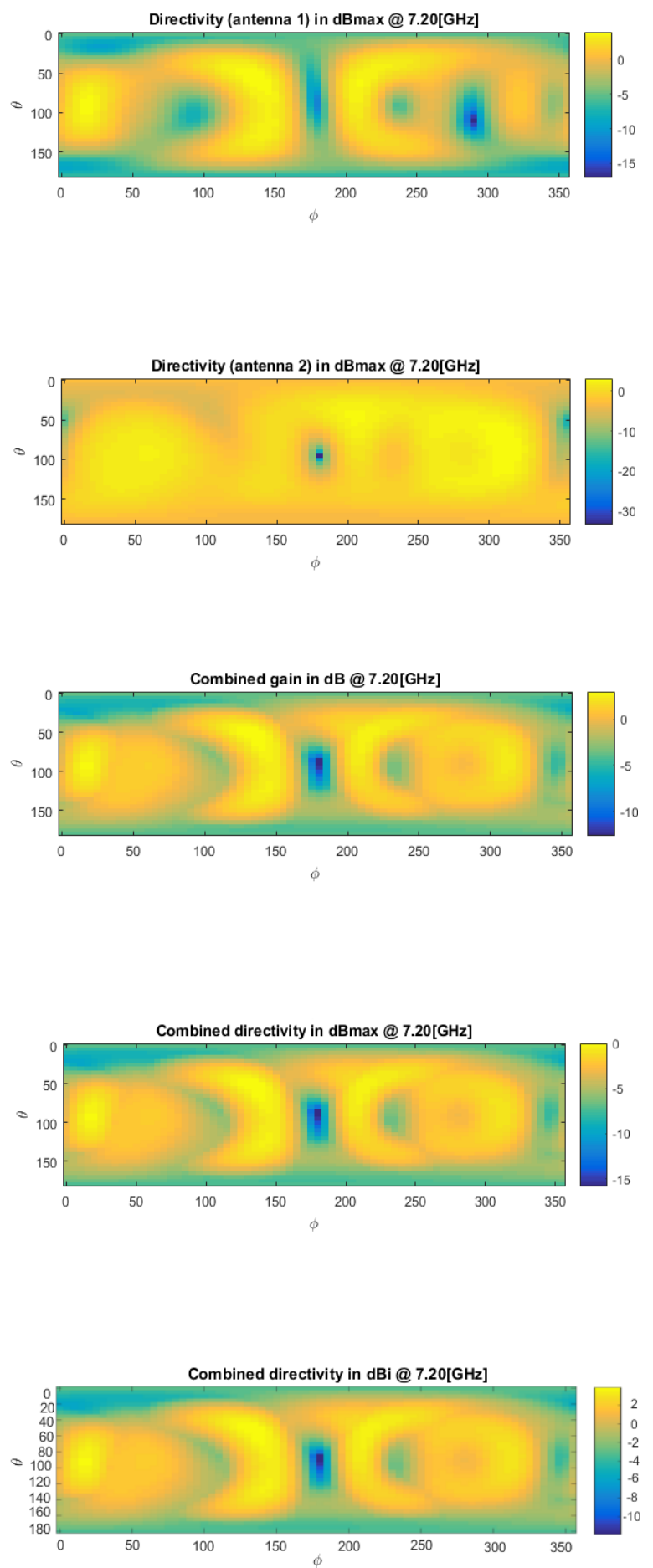

c)

Fig.7 Gain and directivity analysis of UWB diversity antenna a) $6.4 \mathrm{GHz}$, b) $6.8 \mathrm{GHz}$, c) $7.2 \mathrm{GHz}$ 
At $6.4 \mathrm{GHz}$ and $6.8 \mathrm{GHz}$ there is no direction for which the deviation from maximum gain is higher than $10 \mathrm{~dB}$. Hence, omnidirectional coverage is achieved. At $7.2 \mathrm{GHz}$ only $0.9 \%$ of all possible angular directions exhibit higher deviation from maximum gain than $10 \mathrm{~dB}$. The deviation in this region is up to $14 \mathrm{~dB}$.

\section{CONCLUSIONS}

UWB diversity antenna having a size of $41 \mathrm{x} 42.2 \mathrm{~mm}^{2}$ is proposed. To ensure omnidirectional coverage two different radiating elements are designed and pattern diversity is applied. In this way, multipath fading is mitigated. According to the obtained results the proposed antenna can cover 5.45$7.45 \mathrm{GHz}$ frequency band with the mutual coupling and reflection coefficients less than $-15 \mathrm{~dB}$ and $-10 \mathrm{~dB}$, respectively.

\section{REFERENCES}

[1] H. Nikookar and R. Prasad, Introduction to Ultra Wideband for Wireless Communications, Springer Science, 2009.

[2] H. Schantz, The Art and Science of Ultra Wideband Antennas, Artech House, 2015.

[3] D. Valderas, Ultrawideband Antennas: Design and Applications, London: Imperial College Press, 2011.

[4] L. Liu, S. Cheung, T. Yuk and D. Wu, "A Compact Ultrawideband MIMO Antenna," in 7th European Conference on Antennas and Propagation (EuCAP), Gothenburg, 2013.

[5] C. B. Dietrich, K. Dietze, J. Randall Nealy and W. L. Stutzman, "Spatial, Polarization, and Pattern Diversity for Wireless Handhel Terminals," IEEE Transactions on Antennas and Propagation, vol. 49, no. 9, pp. 1271-1281, 2009.

[6] B. C. Wadell, Transmission Line Design Handbook, Artech House, 1991. 